\title{
QUO VADIS THERMODYNAMICS AND THE CITY: A CRITICAL REVIEW OF APPLICATIONS OF THERMODYNAMIC METHODS TO URBAN SYSTEMS
}

\author{
N. FILCHAKOVA, D. ROBINSON \& J.-L. SCARTEZZINI \\ Solar Energy and Building Physics Laboratory, Ecole Polytechnique Fédérale de Lausanne (EPFL), \\ Lausanne, Switzerland.
}

\begin{abstract}
Cities are complex dynamic entities. From a thermodynamic perspective, they represent open systems, constantly importing and exporting energy and matter across their boundaries; the output invariably being less ordered due to irreversible internal processes (dissipation). In analogy to natural ecosystems, the uptake, transport and storage of substances in urban sites as well as their transformation can be considered equivalent to urban metabolism. Apparently inspired by progress that has been made in the study of natural ecosystems, a range of tools based on thermodynamics have recently been applied to better understand this urban metabolism and how it can be optimised. In this paper we review progress that has been made in both domains (i.e. natural and anthropogenic ecosystems). In particular, we consider applications of entropy, energy, exergy and (the quasi-thermodynamic) emergy. We attempt to qualitatively define a seemingly general dichotomy between the explanatory power of a given thermodynamic concept and its calculability. We conclude that whilst thermodynamic considerations indisputably provide a general framework for understanding cities and their evolution, further research is needed for them to become operational representations of urban metabolism and indicators of sustainability. We suggest possible pathways for achieving this.

Keywords: city, entropy, evaluation, open system, sustainable development, thermodynamics.
\end{abstract}

\section{INTRODUCTION}

Unlike natural ecosystems, in which myriad organisms coexist in a process of synergetic exchange such that resource flows are highly circular, in anthropogenic systems like cities they are essentially linear. We (humans) import resources (food, fuel, raw materials), combust and/or process them and export the waste products with almost complete disregard for the environmental consequences. Since half of the global population is currently urbanised, the environmental consequences of this resource inefficiency, such as greenhouse gas emissions and raw material depletion, are alarming. Indeed, with the urban population forecast to increase to three fifths during the next 25 years, the future looks somewhat bleak. It is clearly important then that we better understand this urban metabolism, its adverse consequences and ways in which it can be optimised to minimise these consequences.

In reflecting on the mysteries of life, Schrödinger [1] noticed that rather than dissipate order (or increase entropy) and decay to thermodynamic equilibrium (death), living organisms actually manage to increase or maintain their degree of organisation or order. They achieve this by consuming negative entropy (or rather free energy). More formally, we may say that an organism may be regarded as an open system, in the sense that energy and matter are exchanged across its boundaries. This openness and the presence of flows further imply that the system is also a non-equilibrium one. In other words, although the entropy of isolated physicochemical systems tends to evolve irreversibly to equilibrium $\left(\mathrm{d} S_{\mathrm{i}}>0\right)$, open systems may be maintained in non-equilibrium states. The entropy change $\mathrm{d} S$ in such a system is then $\mathrm{d} S=\mathrm{d} S_{\mathrm{i}}+\mathrm{d} S_{\mathrm{e}}$, where $\mathrm{d} S_{\mathrm{i}}$ corresponds to entropy production due to irreversible internal processes (e.g. human cellular dynamics - for instance, the rapid increase in self-organisation during the early stages of growth from foetus through newborn child to young infant is quite remarkable) and $\mathrm{d} S_{\mathrm{e}}$ corresponds to entropy exchange across the system boundaries 
(we humans consume solid and liquid food, breathe in oxygen and expel carbon dioxide and other waste products). (Indeed, Schneider and Kay [2] suggest that the more energy that is 'pumped' through a system, the greater the degree of organisation that can emerge to dissipate this energy. They go on to suggest that the two processes are inseparable, so if a structure is not 'progressive' enough it will be replaced by a better adapted structure that will use the available energy more effectively.)

Prigogine [3] later formalised these observations, which apply to a large number of systems, creating a new branch of scientific study - modern or far from equilibrium thermodynamics. In 1977, Nicolis and Prigogine [4] suggested that cities may be considered as one such system, noting that a city 'can survive as long as it is the inflow of food, fuel and other commodities and sends out products and waste'. This then is an example of what later came to be known as a dissipative structure, referring to the 'creation and maintenance of organised non-equilibrium states due to dissipative processes' [5].

\subsection{Thermodynamics and the city}

Since Prigogine's initial observation, significant progress has been made in the application of thermodynamic principles to the study of urban development.

One of Prigogine's collaborators, Allen [6,7] pioneered the application of non-linear dynamics to study the spatial dynamics of urban and regional land use. The interactions between different urban actors and their basis for decision-making led to the creation of a functional structure for the city, which supported investigations into the dynamics of its spatio-temporal evolution. Allen's work may be regarded as the forerunner to subsequent (microscopic) studies of urban dynamics based, for example, on cellular automata and agent-based modelling (modelling techniques that have been applied to numerous complex systems, in fields as diverse as (bio)physics, ecology and social sciences). In this respect, Batty [8] has experimented with different neighbourhood rules for mimicking urban growth using cellular automata, and Portugali [9] and Benenson and Torrens [10] have used agent-based models to examine urban relocation and land use change.

More recently, some workers have also investigated the application of thermodynamics to sustainable urban development.

Marchettini et al. [11], for example, discuss two extreme scenarios of an urban system: autarchy and globalisation, arguing that these regimes are not thermodynamically viable. The first approaches what is known as an isolated system, in which interactions and exchanges with the systems environment are inhibited. (To clarify, derived from Greek word for self-governance, autarchic may also be taken to mean independent. We (humans) may be autonomous but we cannot be independent. To live sustainably ourselves, we need relationships with other species; we also need to import energy, e.g. solar energy.) This is known to lead to a fading out of living processes. The second represents an extreme case of an open system that relies on overexploitation of planetary resources. This regime is deemed to be inconsistent with physical laws, since it leads to exhaustion of natural capacity: it is self-destructive (though some may argue that human society is intrinsically self-destructive). Although non-viable, these two extremes may usefully be applied to define the bounds of a liveability space. What remains then is to define a quantitative representation of these extremes as well as of the state of some real urban system that enables it to be placed somewhere between the extremes.

This leads us to the real objective of this paper: to investigate the extent to which the scientific framework of thermodynamics can now be applied to represent urban metabolism in an operational way and the extent to which thermodynamics can be used to help us quantitatively determine urban sustainability (sustainability indicators). For this, we refer to the relatively rich literature on ecosystem theory and to the relatively sparse literature on urban metabolism. As Wilson [12] states, 
'learning about ecosystems and the (fundamentally thermodynamic) laws of their evolution can help us to understand cities in these terms and draw analogies in terms of sustainability between urban and natural habitats'.

\subsection{Quantifying sustainability: general criteria on indicators}

Before embarking on a review of the developments that have been made in these separate but related domains, let us define some general criteria with which we might judge the utility of a particular (quantitative as distinct from qualitative) indicator of sustainability. We suggest the following:

Measurability/calculability: An indicator, by definition, should be quantitative. One must therefore be able to empirically measure or calculate this quantity.

Diagnostic ability: It is important to be able to isolate causal mechanisms for a particular indicator value (or a change in this value). This is particularly relevant for indicators that are based on the aggregation of low-level data (it should be possible to trace back to the appropriate level and variable responsible).

Succinctness (Occam's razor): The indicator should make as few assumptions as possible about the phenomenon in question, and elements which have no observable impact on results should be eliminated.

An indicator should also be constructive: 'it should tell us what to do, not only what not to do. Sustainability policy needs proactive assessment indicators to orient its activities' [13]. We suggest that a good indicator should be constructive and that it must satisfy the above three criteria.

\section{APPLICATIONS OF THERMODYNAMICS TO ECOSYSTEMS}

In his review of the state of the art in ecosystem theory, Müller [14] classifies ecological thermodynamics as 'correct in a very general sense'. He discusses the conceptual framework provided by thermodynamics (the first and second laws, the notion of open systems and the phenomenon of self-organisation). He also briefly reviews the main extrema ecological principles that are based on thermodynamics but were devised in the field of ecology: the non-equilibrium principle [2], the ecological principle of thermodynamics [15], the principle of emergy optimisation (the maximum empower principle) [16], the principles of optimisation of total entropy production and of specific entropy. Müller goes on to state that all these principles (or hypotheses of ecosystem development) lead to similar conclusions:

the more order an ecosystem obtains the lower is its entropy state, the greater is its distance from thermodynamic equilibrium, the higher is the information content, structural complexity, exergy flow and the exergy state and the higher are the total costs for the conservation of complexity and for entropy production.

He sees thermodynamics and principles based on thermodynamics as 'a starting point for a further integration of ecosystem theories' in complement with theories of self-organisation. Thus far, particular progress has been made in respect of exergy and entropy.

\subsection{Exergy}

For exergy (a measure of the ability of a system to perform work), Jørgensen and colleagues [15, 17, 18] proposed the maximum exergy principle, which states that 'an ecosystem strives towards the highest 
possible exergy at the prevailing conditions'. In other words, the exergy content of an ecosystem increases as it evolves. The principle further suggests that if a system receives an input of exergy, it will utilise this exergy to perform work. The work performed: (i) degrades the exergy, dissipating the residue as entropy to the system's surroundings; (ii) moves the system further from thermodynamic equilibrium, reflected in the growth of gradients; and (iii) increases the accumulated energy-mass of the system, representing additional stored exergy [18].

Furthermore, if more than one pathway is offered to depart from equilibrium, the one yielding the highest gradient, the highest throughput (maximum power) and the most storage of exergy under the prevailing conditions, to give the most ordered structure furthest from equilibrium, will tend to be selected.

Exergy thus provides a measure of the fitness of an ecosystem (maximum exergy) and its derivation has helped to deepen our understanding regarding the principles by which natural ecosystems evolve. However, what is not clear is to what extent this framework may be applied to anthropogenic systems in which external influences (e.g. climate) may be compensated for artificially by importing more resources (e.g. energy). Structural adaptations are only required to take place in such circumstances if imports are limited (e.g. we must use a reduced availability of energy more efficiently). The exergy of an urban system may be diagnostically useful, but its calculation, even for natural ecosystems, entails many assumptions; indeed it may not even be feasible to apply this concept in a convincing way to cities.

\subsection{Entropy}

Svirezhev [19] introduced an elegant concept called 'the entropy pump' in application to agricultural ecosystems. In this, the state of a human-modified system is contrasted, by the addition of 'artificial' energy, to that of a natural ecosystem (of corresponding succession type). The total entropy balance of an agro-system is written as:

$$
\frac{\mathrm{d} S}{\mathrm{~d} t}=\frac{1}{T}\left[G P P_{1}+W-G P P_{0}\right]=\sigma,
$$

where $G P P_{0}$ is the gross primary production (J) (primary production is defined as the synthesis and storage by photosynthetic organisms of organic molecules during growth and reproduction and GPP refers to the corresponding carbon that is fixed) of the natural ecosystem, $W$ is the artificial energy (J), including energy and chemical inflows (or anthropogenic pressure), added to the human-modified system of $G P P_{1}$ and $T$ is a mean annual temperature (K). Now if $\sigma>0$, the system overproduces entropy, so the environment is degraded. (Note that in natural ecosystems this overproduction is assumed to be zero as this entropy is sucked out by the solar entropy pump - equivalent to Schrödinger's negentropy.) In principle then, we are able to find the limiting energy load on an agro-system to achieve sustainable yield.

This elegant model allows us to calculate the entropy of a non-equilibrium system under anthropogenic pressure without having to directly calculate the entropy due to individual physical processes. It also provides a measure of the degree of the system's environmental degradation. Svirezhev demonstrates how this model may be implemented in an agricultural context. The challenge in our context is to characterise what might constitute $G P P_{0}$ for an urban settlement. One option might be the hypothetical autarchic system of Marchettini et al. [11], or some thermodynamically viable utopian closed urban system or indeed some natural equivalent to an urban system. The entropy pump hypothesis thus seems to be diagnostically useful, it is succinct and it may well be calculable. 
A very comprehensive (and, to the knowledge of the authors, the single pure) application of non-equilibrium thermodynamics in the non-linear regime to natural (aquatic) ecosystems was developed in a series of works by Mauersberger [20, 21]. He proposed a thermodynamic theory of aquatic ecosystems as a complex system of non-linear differential equations describing the evolution of an ecosystem and its population dynamics. In this, he proposes a complex thermodynamically based framework comprising a set of physical-chemical energy and mass balance equations with rates of biological processes as functions of state variables.

With this work ecology, building on the principles of far from equilibrium thermodynamics, has a sound theoretical basis. However, the practical application and calculation of entropy production, based on this fundamental approach, remains highly difficult. It requires calculation of all thermodynamic flows and forces with subsequent integration over the system volume: a problem which involves too many variables, parameters and uncertainties in natural as well as human ecosystems. This limitation thus renders this fundamental approach rather inappropriate according to our criteria.

\section{APPLICATIONS TO URBAN STUDIES}

In the last decade, a range of attempts have been made to apply thermodynamically inspired (as distinct from truly thermodynamic) principles to analyse the sustainability of urban systems. Of these, emergy and statistical entropy appear to have been most commonly employed.

\subsection{Emergy}

Emergy is available energy of one kind that has been used up to make a product or service [16], expressed in terms of solar energy units (solar energy joules: seJ), using transformities (seJ/J) to convert from conventional energy units $(\mathrm{J})$. These transformities are the key parameters in emergy calculations. When performing emergy analyses, the various stocks and flows are often represented using an energy systems diagram, which helps to structure a potentially complex web of processes. Developed from analyses of natural ecosystems, these diagrams may also qualitatively represent steps in the processes of energy degradation which accompany its transformation - as the primary energy flows from source to sink, so depicting an energy hierarchy [22].

Huang and colleagues have repeatedly applied the emergy method to the analysis of urban systems [23-25]. As part of their more recent work [25] they use the product of urban production $(\mathrm{seJ} / \mathrm{kg}$ ) (which is itself the product of urban area, built floor area, environmental stocks, energy use and population) and a constant calibration coefficient to quantify urban waste generation. This they further define as entropy (seJ/day), which is also interpreted as the 'emergy value of waste flows'. Observing its increase over 40 years, the authors anecdotally conclude that 'urbanindustrial civilisation is vastly accelerating the process of entropy'. Whilst the simplicity of this calculation may be seductive, the proposed entropy has no physical basis. It also seems to be logically flawed. Consider two cities: city 1 is larger than city 2 , likewise the population and the environmental stocks. But city 2 is energy profligate, such that total waste production (or entropy) is similar. However, if more energy is expended to maintain similar thermal gradients (particularly if normalised in terms of built area), then we would naturally conclude that city 2 produces higher entropy than city 1 .

Considering once again our three criteria of a good indicator, then in principle, emergy is calculable, but the centrepiece transformity is shrouded in uncertainty (and sometimes lacks physical meaning). If we take calculability to imply some degree of reasonable precision, then this criterion may not be satisfied. Furthermore, the aggregate results of emergy may submerge the factors which are responsible for a given result - interpretation may be ambiguous. Finally, this method seems to fail 
the test of succinctness. It is a combination of an accounting method (no different in principle to conventional material flow analysis, though in some ways less rigorous: see, e.g. [26]) and a means of conversion of the resultant energy and mass flows, which is not always required and certainly not necessarily in emergy units.

\subsection{Statistical entropy}

Our final port of call concerns the use of statistical or information (as opposed to thermodynamic) entropy in the urban context. These calculations tend to be based on Shannon's equation for the information entropy of a given sequence of binary digits:

$$
H(X)=-\sum_{i=1}^{n} p\left(x_{i}\right) \log _{2} p\left(x_{i}\right)
$$

where $H(X)$ is the information entropy of discrete variable $X$ and $p\left(x_{i}\right)=P\left(X=x_{i}\right)$ is the probability mass function of $X$ [27]. Besides the similarity in the expressions for information entropy and the statistical Boltzmann entropy in physics, there is, in principle, a link between this information theory and thermodynamics, if we define entropy as thermodynamic information. That is, 'all structural changes, fluxes of energy and all matter transfers are coupled with information flows' [14]. Unfortunately, it is not in this respect that information entropy has thus far been applied.

In common with thermodynamics, information theory has been exploited in a more systematic way in ecosystems science, for example, to indicate population/species (or bio-) diversity (see, e.g. indicators of Shannon-Wienner and Margalef in [14]), and as a measure of the diversity of landscape patterns [28]. The latter application is based on analysis of the spatial patterns of maps, the abundance of elements and their diversity, to characterise landscape changes according to the corresponding entropy. High entropy was taken to symbolise disorder (high diversity) and low values indicated high order (low diversity) of the landscape patterns. This method is easily calculable. It expresses overall diversity whilst preserving lower level data, but it does not indicate the driving forces for change (system behaviour is not represented) in landscape order, which limits its diagnostic usefulness. Furthermore, this indicator expresses only the quantity not the quality of landscape diversity. Finally, despite the above drawbacks, it is not obvious how this approach can be meaningfully applied to the urban context in which we are interested, not in spatial diversity per se but in environmental (thermodynamic) degradation. Nevertheless, some attempts have been made.

Referring to open systems, dissipative structures and complex systems theory, Zhang and colleagues $[29,30]$ propose a Shannon-like indicator of urban sustainability (the degree of urban order or health), which they suggest is rooted in thermodynamics:

$$
\mathrm{d} S=-\frac{1}{\ln (j=m)} \sum_{i=1}^{n} \frac{q_{i j}}{q_{j}} \ln \frac{q_{i j}}{q_{j}},
$$

where $q_{i j}$ is one of 42 normalised indicators used to describe urban energy and matter flows, each of which are similarly weighted. The rationale for their inclusion is also unclear. Again this aggregate indicator is calculable, but it is neither succinct nor diagnostically useful.

A similar approach has been employed by Rechberger and Brunner [31] to indicate the entropy (or contaminant dilution) within an industrial (materials processing) context, to which similar criticisms may be levelled. 


\section{DISCUSSION AND CONCLUSIONS}

Reflecting on the various thermodynamically inspired means of evaluation discussed in this paper (for which the key attributes are summarised, according to the criteria established in Section 2.1, in Table 1), we suggest that there is something of a dichotomy between theoretical rigour and practical feasibility.

More specifically, we observe that the calculability of (quasi)thermodynamic expressions is inversely related to their aggregation level, succinctness and diagnostic power. In other words, indicators that are easily calculated do not require strong hypotheses. In the inverse case, quantities requiring strong hypotheses also tend to be difficult to measure, but they have considerably greater explanatory power (Fig. 1).

Efforts to apply thermodynamic (or thermodynamically inspired) concepts to the study of urban metabolism to date have been based on somewhat questionable theoretical foundations, whereas

Table 1: Comparative analysis of a sample of evaluation methods.

\begin{tabular}{|c|c|c|c|}
\hline Indicator & Measurable/calculable & Traceable & Succinct \\
\hline \multicolumn{4}{|l|}{ As applied in ecology } \\
\hline Exergy & Medium & High & High \\
\hline \multicolumn{4}{|l|}{ Entropy } \\
\hline Classical TD & Medium & High & High \\
\hline Modern TD & Low & High & High \\
\hline \multicolumn{4}{|l|}{ As applied in urban studies } \\
\hline Emergy & Medium/high & Medium/high & Medium/low \\
\hline Statistical (quasi)entropy & High & Low & Low \\
\hline
\end{tabular}

Succintness/

thermodynamic basis

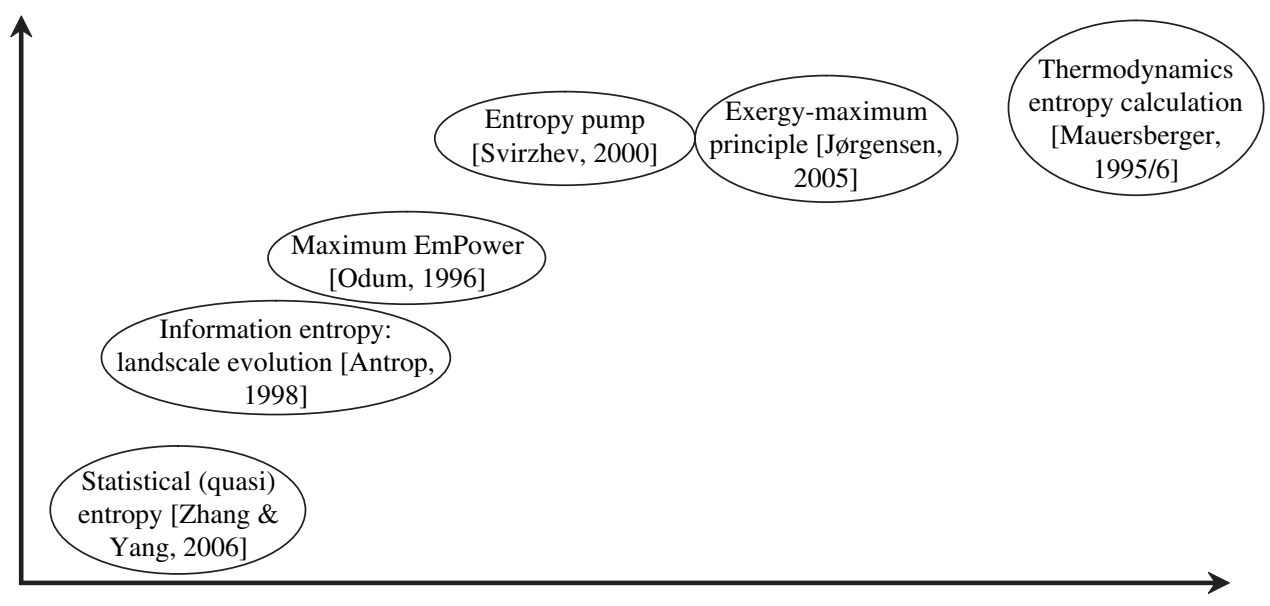

Difficulty of calculation

Figure 1: Qualitative categorisation of a sample of evaluation methods. 
many of the studies from the field of ecology are rigorous and offer good potential for application to urban studies. This is particularly the case with respect to Svirezhev's entropy pump hypothesis and also (though perhaps to a lesser extent) to Jørgensen's exergy analysis.

These principles should help us to understand and evaluate urban metabolism, though not necessarily to encapsulate it at a functional level through mathematical models. As Tiezzi notes, this metabolism is 'both complex and complicated, and therefore not completely determinable' ([32], p. 92). Perhaps the thermodynamically influenced models of the dynamics of urban growth (Section 1) will provide food for thought here?

\section{ACKNOWLEDGEMENTS}

The financial support for this work from National Research Programme 54 of the Swiss National Science Foundation is gratefully acknowledged.

\section{REFERENCES}

[1] Schrödinger, E., What is Life? Cambridge University Press: Cambridge, 1944.

[2] Schneider, E.D. \& Kay, J.J., Complexity and thermodynamics. Futures, 26(6), pp. 626-647, 1994.

[3] Prigogine, I., Introduction to Thermodynamics of Irreversible Processes, Wiley: New York, 1967.

[4] Nicolis, G. \& Prigogine, I., Self-Organization in Nonequilibrium Systems, Wiley-Interscience: New York, 1977.

[5] Kondepudi, D. \& Prigogine, I., Modern Thermodynamics. From Heat Engines to Dissipative Structures, John Wiley \& Sons: Chichester, 1998.

[6] Allen, P., Towards a new synthesis in the modelling of evolving complex systems. Environment and Planning B, 12, pp. 65-84, 1984.

[7] Allen, P., Cities and Regions as Self-Organising Systems: Models of Complexity, Gordon and Breach Science Publishers: Amsterdam, 2003.

[8] Batty, M., Cities and Complexity, MIT Press: Cambridge, 2005.

[9] Portugali, J., Self-organising cities. Futures, 29(4/5), pp. 353-380, 1997.

[10] Benenson, I. \& Torrens, P., Geosimulation: Automata-Based Modelling of Urban Phenomena, Wiley: London, 2004.

[11] Marchettini, N., Pulselli, F.M. \& Tiezzi, E., Entropy and the city. WIT Transactions on Ecology and Environment, 93, pp. 263-272, 2006.

[12] Wilson, A.G., Ecological and urban systems models: some explorations of similarities in the context of complexity theory. Environment and Planning A, 38, pp. 633-646, 2006.

[13] Newman, P., Environmental impact of cities. Environment \& Urbanization, 18(2), pp. 275-295, 2006.

[14] Müller, F., State-of-the-art in ecosystem theory. Ecological Modelling, 100, pp. 135-161, 1997.

[15] Jørgensen, S.E. \& Svirezhev, Y.M., Towards a Thermodynamic Theory for Ecological Systems, Elsevier: Amsterdam, 2004.

[16] Odum, H.T., Environmental Accounting, Emergy and Decision-Making, Wiley: New York, 1996.

[17] Jørgensen, S.E., Costanza, R. \& Li, F.-L. (eds), Handbook of Indicators for Assessment of Ecosystem's Health, CRC Press: Florida, 2005.

[18] Patten, B.C. \& Jørgensen, S.E. (eds), Complex Ecology: The Part-Whole Relation in Ecosystems, Prentice Hall: Englewood Cliffs, NJ, 1995.

[19] Svirezhev, Y. M., Thermodynamics and ecology. Ecological Modelling, 132, pp. 11-22, 2000. 
[20] Mauersberger, P., Entropy control of complex ecological processes. Complex Ecology: The Part-Whole Relation in Ecosystems, eds B.C. Patten \& S.E. Jørgensen, Prentice Hall: Englewood Cliffs, NJ, 1995.

[21] Mauersberger, P., From a theory of local processes in aquatic ecosystems to a theory at the ecosystem scale. The Science of the Total Environment, 183, pp. 99-106, 1996.

[22] Brown, M.T., Odum, H.T. \& Jørgensen, S.E., Energy hierarchy and transformity in the universe. Ecological Modelling, 178, pp. 17-28, 2004.

[23] Huang, S.-L., Lai, H.-Y. \& Lee, C.-L., Energy hierarchy and urban landscape system. Landscape and Urban Planning, 53, pp. 145-161, 2001.

[24] Huang, S.-L. \& Chen, C.-W., Materials flow analysis and emergy evaluation of Taipei's urban construction. Landscape and Urban Planning, 63, pp. 61-74, 2003.

[25] Huang, S.-L. \& Chen, C.-W., Theory of urban energetics and mechanisms of urban development. Ecological Modelling, 189, pp. 49-71, 2005.

[26] Baccini, P. \& Bader, H.-P., Regionaler Stoffhaushalt, Spektrum Verlag: Heidelberg, 1996.

[27] Shannon, C.E., A mathematical theory of communication. The Bell System Technical Journal, 27, pp. 379-423, 1948.

[28] Antrop, M., Landscape change: plan or chaos? Landscape and Urban Planning, 41, pp. 155-161, 1998.

[29] Zhang, Y. \& Yang, Z., Measurement and evaluation of interactions in complex urban ecosystem. Ecological Modelling, 196, pp. 77-89, 2006.

[30] Zhang, Y., Yang, Z. \& Li, W., Analyses of urban ecosystem based on information entropy. Ecological Modelling, 197(1-2), pp. 1-12, 2006.

[31] Rechberger, H. \& Brunner, P.H., A new, entropy based method to support waste and resource management decisions. Environ. Sci. Technol., 36, pp. 809-816, 2002.

[32] Tiezzi, E., Steps towards an Evolutionary Physics, WIT Press: Southampton, UK, 2006. 